\title{
A NEW THERAPEUTIC APPROACH TO VULVODYNIA: PULSED RADIOFREQUENCY OF THE IMPAR GANGLION CAN ALLIEVIATE CHRONIC PELVIC PAIN; A CASE SERIES
}

\author{
A Kelly, B Bonet \\ Leighton Hospital, Crewe, UK
}

Vulvodynia is a common, chronic, disabling condition causing significant morbidity and is detrimental to the patients' quality of life ${ }^{1}$ Pain is a primary factor in this morbidity There is no agreed approach to pain management in idiopathic, neuropathic vulvodynia

Background

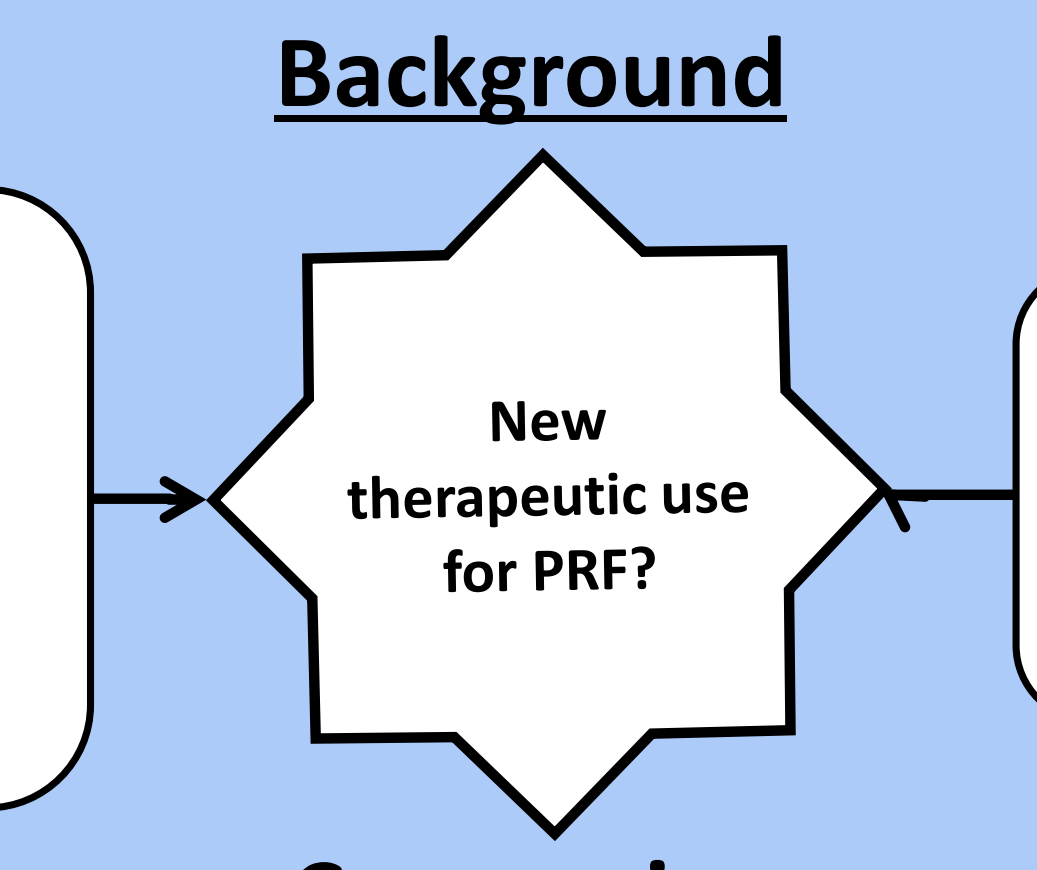

Case series

- We present two cases in which detrimental vulvodynia was successfully treated with Pulsed Radiofrequency

- Both patients had had previous unsuccessful dermatological treatment, including lidocaine patches

- Both had previously had unsuccessful pudendal nerve block

\section{The Treatment}

- Pulsed Radiofrequency (PRF) to Impar Ganglion

- One six minute session of PRF, at $45 \mathrm{~V}$ and $5 \mathrm{~Hz}$

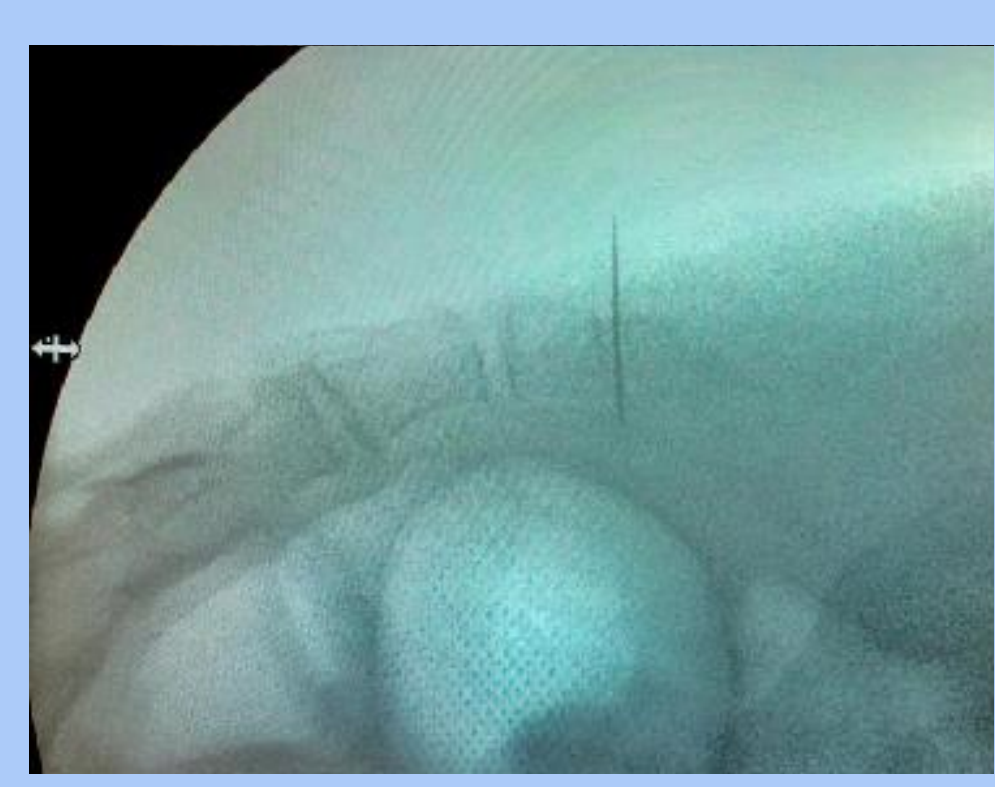

PRF of the Impar Ganglion

\section{Case 1}

45 year old woman

18 month history of vulvodynia

Unsuccessful treatment pharmacologically

Multiple emergency department attendances

8/10 on VAS score, preventing intercourse

One PRF intervention to the Impar Ganglion.

After treatment: VAS score of 0 . Stopped all analgesia,

Pain free 12 months later

\section{Case 2}

65 year old woman

4 year history of vulvodynia

Unsuccessful treatment pharmacologically

Extremely disabling for patient

$10 / 10$ on VAS score, preventing day to day activities including walking

One PRF intervention to the Impar Ganglion.

After treatment: VAS score of 2. Reduction in all pharmacological analgesia.

\section{Discussion}

Vulvodynia can be difficult to manage, and can lead to significant morbidity for the person suffering

We have shown the successful use of PRF in the management of this chronic pelvic pain, allowing significant reduction in the pharmacological therapy, and improvement in the quality of life of these two women

Only one six minute PRF session was needed to achieve this symptom relief Importantly, PRF is well tolerated intervention, with a minimal side effect profile, already in use in a wide range of patients around the world

This novel analgesic approach gives clinicians and patients a new analgesic option in treating the disabling pain of vulvodynia

\section{Learning Point}

This case demonstrates Pulsed Radiofrequency to the Impar Ganglion to be a new approach to managing vulvodynia when conventional management has failed

References:

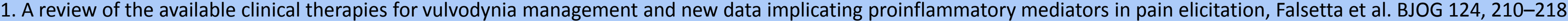
10.1111/1471-0528.14157.

2. Vanneste T, Lantschoot AV, Van Boxem K, Van Zundert J. Pulsed radiofrequency in chronic pain. Curr Opin Anaesthesiol. 2017;30(5):577-582. 